\title{
Collaborative Healthcare Remodelling through Sequential Simulation (SqS): A Patient and Front-line Staff Perspective
}

Sharon-Marie Weldon, Roger Kneebone, Fernando Bello

\section{Corresponding author:}

Sharon-Marie Weldon

Senior Research Officer

Centre for Engagement and Simulation Science

Imperial College London

3rd Floor Chelsea and Westminster Hospital (Academic Surgery)

369 Fulham Road

London SW10 9NH

s.weldon@imperial.ac.uk

Tel: +44 (0) 2033155468

Roger Kneebone

Professor of Surgical Education and Engagement Science

Centre for Engagement and Simulation Science

Imperial College London

Dept. Surgery and Cancer

London, UK

Fernando Bello

Reader in Surgical Computing and Simulation Science

Centre for Engagement and Simulation Science

Imperial College London

Dept. Surgery and Cancer

London, UK

\section{KEYWORDS}

Sequential Simulation (SqS)

Co-production

Simulation

Patient Involvement

Healthcare re-modelling

Word count: 3431 


\section{ABSTRACT}

Background: The Department of health funded an initiative to pioneer new approaches that would create a more integrated form of care.

Local problem: In order to receive funding, local Clinical Commissioning Groups were required to engage a range of stakeholders in a practical approach that generated the development of an integrated model of care.

Intervention: Two Sequential Simulation (SqS) workshops comprising 65 and 93 participants, respectively, were designed using real patient scenarios from the locality, covering areas of general practice, community health and adult social care. Workshops were attended by a diverse group of stakeholders. The first workshop addressed current care pathways and the second modelled ideal care pathways generated from the data obtained at the first workshop.

Methods: Discussions were captured through video-recording, field-notes and pre and post questionnaires. Data was collated, transcribed and analysed through a combination of descriptive statistics and thematic analysis.

Results: The questionnaires revealed that attendees strongly agreed that they had had an opportunity to contribute to all discussions and raise questions, concerns and ideas (100\%). Pre and post knowledge of current and new models of care was vastly improved. The opportunity to share information and to network was valued, with the SqS approach seen as breaking professional barriers (100\%).

Conclusion: Simulation can be used as a tool to engage stakeholders in designing integrated models of care. The systematic data collection from the diverse ideas generated also allows for a muchneeded 'ear' to those providing the solutions, as well as a legitimate and balanced perspective.

\section{What is already known on this subject?}

\section{What this paper adds}

- The development of an integrated model of care requires front-line staff, patients, lay members and managers to engage.

- The difficulty in bringing various stakeholders together (who use different terminology and therefore speak different languages) is well known.

- In order to engage a range of stakeholders effectively, boundaries need to be broken down, experiences shared and mutual understanding built.

\section{What this study adds}

- Simulation, and in particular Sequential Simulation (SqS), can be applied as a tool to inform, design and operationalize integrated care, breaking down boundaries and building mutual understanding between stakeholders.

- Further studies of this approach are needed in order to understand the benefits that SqS can provide in terms of collaboratively remodelling healthcare. 


\section{INTRODUCTION}

Commencing in 2013, the Department of health (DH) funded an initiative that spanned fourteen boroughs across England designed to pioneer new approaches that would create a more integrated form of care ${ }^{(1)}$. Integrated care aims to address system fragmentation in order to improve the patient experience, whilst ensuring healthcare efficiency and value for money ${ }^{(2)}$. In order to receive funding, the DH required local Clinical Commissioning Groups (CCGs) to develop practical approaches that engaged front-line staff, patients, lay members and managers, in the development and visualisation of an integrated model of care ${ }^{(1)}$.

The difficulty in bringing various stakeholders together (who use different terminology and therefore speak different languages) is well known ${ }^{(3)}$. Longest ${ }^{(4)}$ state:

'Communication between people who use different terminology can be ineffective simply because people attribute different meanings to the same words. When a message both is complex and contains terminology that is unfamiliar to the receiver, it is particularly likely that misunderstanding will occur. This contextual barrier often inhibits communication not only within health programs but also between health programs and many of their external stakeholders' p.213

The dilemma encountered by the CCG's was how to authentically engage a variety of stakeholders in the development and visualisation of an integrated model of care. Although there has been a drive towards more stakeholder involvement and, in particular, Public and Patient Involvement (PPI) over recent years ${ }^{(5)}$, these approaches rely on theoretical discussions and documentation, accentuating the stakeholder communication barrier. Bourdieu ${ }^{(6)}$ argues that through this process situations are created in which health related knowledge obtained through formal education is more highly valued than other forms of formal or informal knowledge held by stakeholders. The symbolic and cultural capital that healthcare professionals possess accentuates this issue further. Therefore, contributions by other stakeholders have the potential to be downplayed when in contrast to existing capitals and even shut out altogether when medical language is used. Elliott and Williams ${ }^{(7)}$ propose that, in order to engage a range of stakeholders effectively, boundaries need to be broken down, experiences shared and mutual understanding built. They suggest 'the development of initiatives, which may require professionals to engage in deliberations outside their traditional professional terrains both intellectually and sometimes physically' p.1113.

In light of these dilemmas, we propose simulation as a means to address these issues, whilst simultaneously broadening simulations application, and, in particular, Sequential Simulation (SqS) ${ }^{[8]}$.

Simulation has been traditionally used as a training tool for healthcare practitioners with particular clinical objectives. However, Sequential Simulation (physically simulated trajectories of care) is a concept that we developed in order to address multiple training requirements from a patient's perspective. The overall concept takes elements of a patient's care pathway that are developed into a scenario based simulation using real clinicians and simulated patients in order to create a simulated experience from a patient's perspective. This concept was then further adapted to be used as a central focus for generating engagement and involvement of a variety of stakeholders in the development of a new integrated model of care. The SqS model has been designed through 
empirical data. It is objective based, thus ensuring each step in the pathway highlights the issues at hand. To date, SqS has been used for training healthcare professionals and workers holistically ${ }^{(9,10)}$, engaging young people with Multi-disciplinary teams ${ }^{(11)}$, engaging patients in research around new diagnostic interventions ${ }^{(12)}$, and designing new models of care.

The aims of this project were to utilise the SqS concept to inform, design and operationalise integrated care from a bottom up approach in order to fully engage and involve all stakeholders. This paper has two objectives: 1) To describe the methods and results of the engagement through sequential simulation approach, as well as their contribution to collaborative healthcare remodelling. 2) To describe the research methods and results used to understand this new application of simulation and its potential.

\section{METHODS}

In late 2014, an urban Clinical Commissioning Group and our research team at Imperial College Centre for Engagement and Simulation Science (ICCESS) collaborated to undertake two sequential simulation (SqS) workshops based around the development of a new integrated model of care for a Greater London Borough.

The first workshop comprised three scenarios based on real patient stories that were designed through the SqS model and subsequently sequentially simulated, covering areas of general practice (Raj's story), adult social care (John's story) and community health (Navneeta's story). Each simulated scenario was followed by facilitated table discussions (8-10 people per table) to identify issues in the current system, solutions to these issues and red tape that prevented solutions. A clinical group facilitator captured data from the discussions. Further discussions were held at a whole group level (65 participants) [Figure 1] and captured through video-recording and field-note taking. Pre and post questionnaires were also completed before and after each workshop that addressed the application of simulation in this format. All the data from the first workshop was collated and analysed through a combination of descriptive statistics and thematic analysis. The remodelling data was split into the associated story issues, solutions and red tape. Themes were identified in the issues data and corresponding solutions were compiled under the identified themes. Based on this information, three new 'ideal world' scenarios were designed using the SqS model from the data collected.

\section{Figure 1. Workshop 1 attendance grouped by role (65 attendees)}

The second workshop format commenced by replaying a video of the first workshop SqS scenarios, followed by a run through of the analysed data and then the SqS of the new co-designed scenarios. The same format as the first workshop of smaller facilitated table discussions (8-10 per table) and larger group feedback sessions (Figure 2) then followed (93 participants). Data was captured in the same process as for the first workshop and analysed in the same way.

\section{Figure 2. Workshop 2 attendance grouped by role (93 attendees)}


The following sections depict one of the three scenarios (Navneeta's story), run across both workshops, as an example of the methods used and the results obtained.

Figure 3 describes the care pathway of Navneeta simulated during workshop 1. The SqS scenario was designed using a real patient's story entered into the SqS model in order to ensure it met the required objectives.

\section{Figure 3. A diagram of Navneeta's 'current' Sequentially Simulated story}

After Navneeta's sequential simulation, the attendees were split into mixed professional/patient groups of 8-10 and were required to discuss: What was wrong with the current system? (e.g. where are the gaps? Duplication, fragmentation in the current system?); What should be changed?; What are the red tape issues? (e.g. not in my job description, or 'the process does not allow me to do xyz and/or issues around organisational culture - how people view themselves, their role and their organisation, difference between sectors/professions); What resources are needed for this? (e.g. time, manpower, money, training); What communication systems are needed (e.g. what do people need or want to know? and/or what IT changes would make a step change in how to integrate and improve their work?); and any other issues.

Each group was provided with a facilitator from a health and social care background who took detailed notes and ensured the discussions addressed the required questions. After 45 minutes, the attendees were brought back into the larger group to feedback the key points of what they had discussed. This feedback was video-recorded (and later transcribed) and further notes were taken for subsequent thematic analysis.

From the above information retrieved, a new scenario was derived of what could have happened to Navneeta based on the attendees' suggested solutions. In order to provide consistency for the SqS and to ensure the new approach is not too 'idealized', the 'ideal' scenario was commenced at the same place the original scenario started. Figure 4 depicts the new 'ideal' simulated scenario.

Figure 4. A diagram of Navneeta's Sequentially Simulated 'ideal' story

After the SqS, the attendees were again split into mixed professional groups of 8-10 and this time were required to discuss: What works in the new system? (MDT / Care Plan / Care Coordination / Empowerment and Self-Help); What doesn't work?; What might prevent the new system from working as intended and what do we do about this?; What's missing from 'our' model of care?; Is there a smarter way of delivering person-centred care for Navneeta?; What resources are needed for this? (e.g. time, manpower, money, training); What might I need to do differently in my role to make these changes happen?; What training or support might I need to do my work in this scenario?; How do I and my colleagues in other organisations work as a team?; and any other issues (e.g. red tape /organisational / culture differences). Each group was again provided with a facilitator from a health and social care background who took detailed notes and ensured the discussions answered the required questions. The facilitators were also asked to consider the following: 
- Re-orientate the team around a culture of delivering a seamless, person-centred care to the patient / service user / carer, regardless of which organisation is providing that part of the service

- Check the effectiveness of the care plan, particularly from the front-line staff perspective

- Give some thought to how we treat cross-border issues

- Agree on the composition of the MDT - persons in the MDT must be the people who deliver the actual care to the patient or carer

- If there is a core and satellite team model, how should the availability of MDT members best be utilised

- Agree the roles and responsibilities of MDT members and the skills and behaviours needed

- Decide where MDT members are to be physically located and how they are to come together in the MDT

- Decide how often the MDT will meet, the range of conditions and the numbers of patients to be seen

- Agree how health and social care assessment processes can be integrated.

After 45 minutes, the attendees were brought back into the larger group to feedback the key points of what they had discussed. This feedback was video-recorded (and later transcribed) and further field notes were taken by the research team.

\section{Ethical Considerations}

Ethical approval was obtained from the Imperial College Research Ethics Committee (ICREC Reference: 14IC2251). Informed consent was obtained from all participants.

\section{RESULTS}

\section{Remodelling data results}

All the data collected from SqS Navneeta's story discussions was collated and divided into: Identified solutions, suggested solutions and red tape issues. The data was then further categorized under main theme headings (see Table 1 for a table format of this data). Under the main theme headings, further sub headings were identified and categorized and corresponded to both the identified issues and suggested solutions categories. The following is an overview of the issues identified and suggested solutions to these issues. Interestingly, in all categories many more solutions were identified compared with issues.

\section{Workshop 1 data summary of Navneeta's care pathway}

It was identified that there was a lack of consistency, training, time, knowledge and power for carers to flag up issues, even though they are in a privileged position to do this. Communication between health and social care providers needed to be more coherent and mechanism needed to be put in place to support this. 
Many voluntary services and re-enablement service are available to empower patients to become more independent and less isolated. However, mechanisms to identify who needs these services are limited. Bereavement is a good example of a change in circumstances that can cause individuals to become isolated and lonely and links should be made to funeral services and religious groups to work out how to flag these happenings. Professionals need to be better at not medicalising everything and instead recognising the social issues that cause the medical problems.

Other system breakdowns include the link between 999 calls, paramedics and primary care. Information between these services needed to be shared to prevent unnecessary admissions. A care coordinator, A\&E case worker or integrated care team is needed to join the dots in these cases. 
Table 1. Categorised thematically analysed data of Navneeta's current story

\section{IDENTIFIED ISSUES}

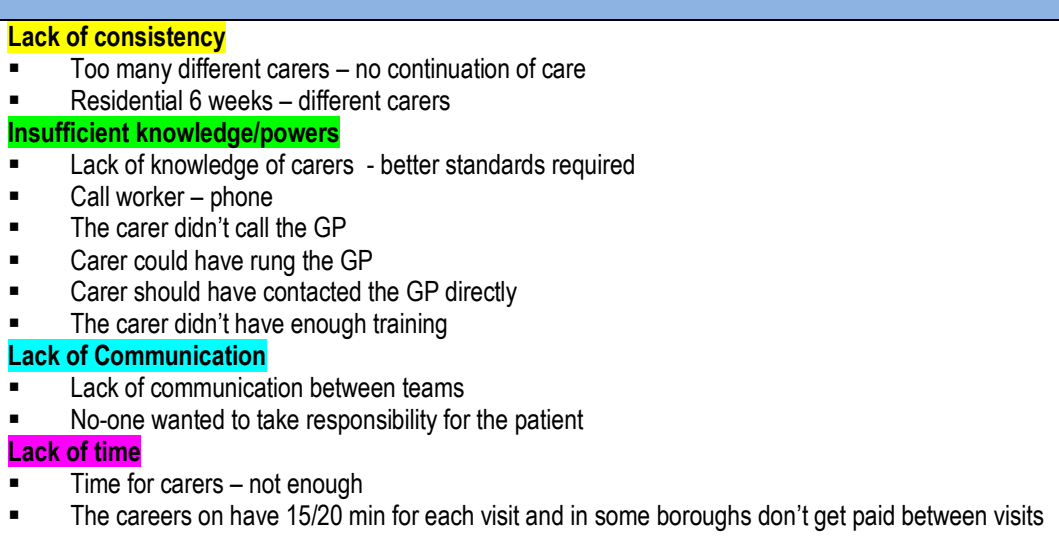

TRAINING KNOWLEDGE \& EMPOWERIENT

Lack of consistency

- Continuity for carers - acknowledgement of roles

adults

- Telephone support/telehealth

- Prompts for carers - is your pt. showing signs of depression?

- Carer needed more freedom to act on her behalf

- Increase pay of London allowance to encourage carers

- $\quad$ Same carers - bluebird system

Insufficient knowledge/powers

- No dumping - shared care

- Training for carers - complex care

Lack of Communication

- Multiple hospital admissions should be an indicator

- Care-coordinator in place

- Increased empowerment of carers to be proactive with support/care referrals - workforce education regarding vulnerable

- Raising awareness of pharmacists

- Redistribute

- Better communication between hospital and the GP

- Every person in a care team should proactively manage a patient

- The trust should use more voluntary services

- Bank of information for all professions

- There should be better communication between the carer and GP and hospital and $G P$

- Multi-agency, data analysis of frequent hospital admissions

Lack of time

SOLATION

\section{Loneliness}

Lonelines

Isolation of patient
$\quad$ Lack of human contact

- Loneliness

Bereavement

- We need to find ways of stopping medicalising everything when it is actually a social isolation and potential a loneliness bereavement issue

Behaviour

Learnt behaviour and dependence

- Better use of befriending/voluntary services

- Resources for loneliness and social isolation

- Friend service

- Age concern

- Extra sheltered care - activities available

\section{Bereavemen}

- Engage with people like funeral directors

- Religious groups

- Time and face to face contact needed

Behaviour

- Try to identify the assets that the pt. can give back to the community

SERVICE BREAKDOWN

System communication mechanisms
$\quad 999$ services - co-ordinated working - not pushing to acute care! - improved pathways

- System failure

- $\quad 999$ - taking responsibility - GP

System communication mechanisms

- Discharge note to GP?

- Social worker? 
- Not one of 5 people who saw the pt. took responsibility

- If her husband died and no one alerted the pharmacy. Where do we link that up so that the pharmacy is made aware? It there was a way of following trough someone's bereavement. They get quite a lot of care for the first 8 weeks and then there is a gap.

- No communication between the implementers of IT systems and the people who use the systems

Reactive approach rather than proactive approach

- Systems need to be put in place before they go home

- It took a while formed unable to leave the pt. at hor the consultant to realise this a pattern and to contact her GP

Bereavement

- Poor bereavement support/social support

- It wasn't just bereavement she was missing

Appropriate support

- She didn't have a nursing need more in the remit of social care

- Lack of information about pills

- Signposting of support needs to be better

Simplify the system

Teams aligned together

Improved triage of emergency calls

- Sharing of information between services

- Streamline communication with hospital

How can communication processes be joined up?

- One page sheets for paramedics to know what are 'normal' parameters

- Social workers within general practice

- GP protocol communication, or careers; floating (?) support

Yellow pages of services

Reactive

Care navigator service

- A\&E case worker

Red/amber/green volunteer services

Demand manager

- Integrated response team was needed

SUPPOR

Culture needs to be changed re. expecting a home visit

\section{Bereavement}

- Bereavement support from GP services

Proactive bereavement support process

- Duty Dr. system can help with conversations (Dr rings you back) and many help with someone to talk to

- Bereavement is the key to this and many patients

\section{Appropriate support}

\section{Promote independence}

Voluntary organisation

Re-enablement services are importan

- Pharmacist make a call to the GP

Support group activities not joined up, e.g. Healthy in Hounslow Event

- Supported housing network, would be hugely beneficial. They notice if the social interaction $n$ not taking place.

Should we be working with funeral directors to improve help for people who have been bereaved or faith communities?

- Need for one person to be engaged to 'care' for the patient to be able to support

- Care support workers/carers/care-navigators - complicated!

\section{- Restrictions due to capacity}

We rely on carer feeding back issues but not mechanism for this

- The whole system isn't constructed in a way to identify if patients are accessing the services.

- Who co-ordinates/navigates care - not clearly articulated

Professionals need to know services available

- Lack of information about services no longer in existence

What can GP do?

- Who should care navigate

- Current bereavement service will not visit pts. In their houses

Hounslow needs a service data base like the voluntary support service database - Needs to be up-to-date, comprehensive, and searchable by religion, ethnic, geographic location, etc. 


\section{Workshop 2 data summary of Navneeta's 'ideal' suggested solutions}

Using an Integrated Community Response Service (ICRS), a care coordinator and a joined-up approach with paramedics was identified as a good approach by attendees. However, attendees felt that the approach could have worked better if pharmacists and the voluntary sector would have been involved and the root cause (bereavement) would have been identified earlier on. They also felt that the learned behaviour of calling 999 had to be addressed too. Still missing from the scenario was a bereavement trigger mechanism that would identify individuals who had a recent change in circumstances due to bereavement. If this was available, an assessment could be made early on to identify the patients' needs and create a care plan. In order to achieve this and a more joined up approach, attendees felt a proper IT structure accessible to all was paramount (see Table 2 for catergorised data). 
Table 2. Categorised thematically analysed data of Navneeta's 'ideal' story

\begin{tabular}{|c|c|c|c|c|c|}
\hline \multicolumn{6}{|c|}{ What works in the new system } \\
\hline $\begin{array}{l}\text { Services } \\
\text { Saved a hospital admission } \\
\text { Increased consistency } \\
\text { Responsible } \\
\text { Accessed the services available } \\
\text { Referral to ICRS-direct from LAS ) Primary } \\
\text { care MH, strategies for panic attacks) } \\
\text { Non-medical prescribing } \\
\text { Access to twilight nursing service } \\
\text { Avoided hospital admission } \\
\text { Admission avoided }\end{array}$ & $\begin{array}{ll}\text { Carers } \\
\text { : } & \text { Carer was empowered } \\
\text { : } & \text { More pro-activity to carers - referring }\end{array}$ & \multicolumn{2}{|c|}{$\begin{array}{l}\text { Joined up } \\
\text { Appropriate referral - vital } \\
\text { Joined up better, but not perfect - pt } \\
\text { not empowered - socialisation } \\
\text { More joined-up thinking } \\
\text { Community matron involved } \\
\text { coordinator }\end{array}$} & & $\begin{array}{l}\text { Other comments } \\
\text { Age concern less isolated }\end{array}$ \\
\hline \multicolumn{3}{|l|}{ What didn't work } & \multicolumn{3}{|l|}{ What was missing? } \\
\hline \multicolumn{3}{|c|}{$\begin{array}{l}\text { Support } \\
\text { Still not tackling the essence of the problem } \\
\text { Root cause of anxiety not tackled, no bereavement service } \\
\text { Transport for befriending - potential to go out- voluntary service } \\
\text { Becoming more dependent - no obvious plan } \\
\text { Bereavement still an issues } \\
\text { Poster in house if further help needed } \\
\text { Roles } \\
\text { Ghere referrals are coming from changed - community referral. } \\
\text { Patient grours directive - pharmacists trained to prescribe on feedback to GP - e-mail } \\
\text { Paramedic - fully trained and empowered } \\
\text { Rehab - extend to physio - pt getting out and about } \\
\text { Communication between care agency and community matron } \\
\text { Healicealth/social services separate funding needs to be more seamless. } \\
\text { Real world scenario - age concern (waiting list) } \\
\text { 07:00-19:00 hours - } 7 \text { days a week. } \\
\text { Calling } 111 \text { - produce automatic } 999 \text { callout } \\
\text { The use of community pharmacy was a good step forward but it could have been taken further and there } \\
\text { are community pharmacists in other areas who actually prescribe and dispense the trimethoprim for a UTI } \\
\text { infection so you don't actually have a referral from a GP it's done under what's now as a PGD patient group } \\
\text { directive, the information is then sent by e-mail to the NHS.net system to the GP so they are aware in real } \\
\text { time that this antibiotic has been dispensed but it would have saved a lot of time } \\
\text { I think the root cause wasn't mechanised by the care and I think the care package wasn't right because it } \\
\text { hadn't been staff matched, they could have easily found out the root cause of why she was anxious, you } \\
\text { know her husband had just died, and it was very simple actually but no one really recognised that it was } \\
\text { still going on reactive as supposed to proactive }\end{array}$} & \multicolumn{3}{|c|}{$\begin{array}{l}\text { Support } \\
\text { Bereavement counselling } \\
\text { - } \text { Re-enablement } \\
\text { Supporting people service } \\
\text { Addressing the mental health issues } \\
\text { Generation of 'support plan" } \\
\text { Psych input for anxiety } \\
\text { Bereavement support } \\
\text { Needed befriending/companionship } \\
\text { - } \text { Family not identified } \\
\text { Staff matching for cultural needs } \\
\text { Social worker to take a voluntary list and pitch 'befriender' } \\
\text { Pt. wanted companionship - she needs volunteers/home visitors } \\
\text { Council (on register of death) contact GP within } 48 \text { hours } \\
\text { Fit link line } \\
\text { Directory } \\
\text { Difficulties and caring society - need to empower patient and knowledge - how they access the knowledge? } \\
\text { Learned pattern/sick role needs to be broken } \\
\text { Link line (emergency button) - carers } \\
\text { Services understanding of her loneliness and isolation } \\
\text { - Passing on information/highlighting problematic systems } \\
\text { Notification when registering death to LA - to do a home visit within } 3 \text { months - check fire/fire alarms/slip, trip, } \\
\text { falls/heating - signposting to schemes } 0 \text { i.e. come and repair handyman. } \\
\text { Ask permission over } 75 \text { years - thread through system } 1 \text { - GP } \\
\text { COPD management } \\
\text { Is her home situation appropriate? Does she need to be in sheltered accommodation - social care not explored } \\
\text { Voluntary sector (but commissioned) rapid response? } \\
\text { Staff remain rushed even in second scenario } \\
\text { Higher salary for carers training } \\
\text { Carers taking responsibility } \\
\text { Funding - personal budgets - pt. and carers had assistance }\end{array}$} \\
\hline
\end{tabular}




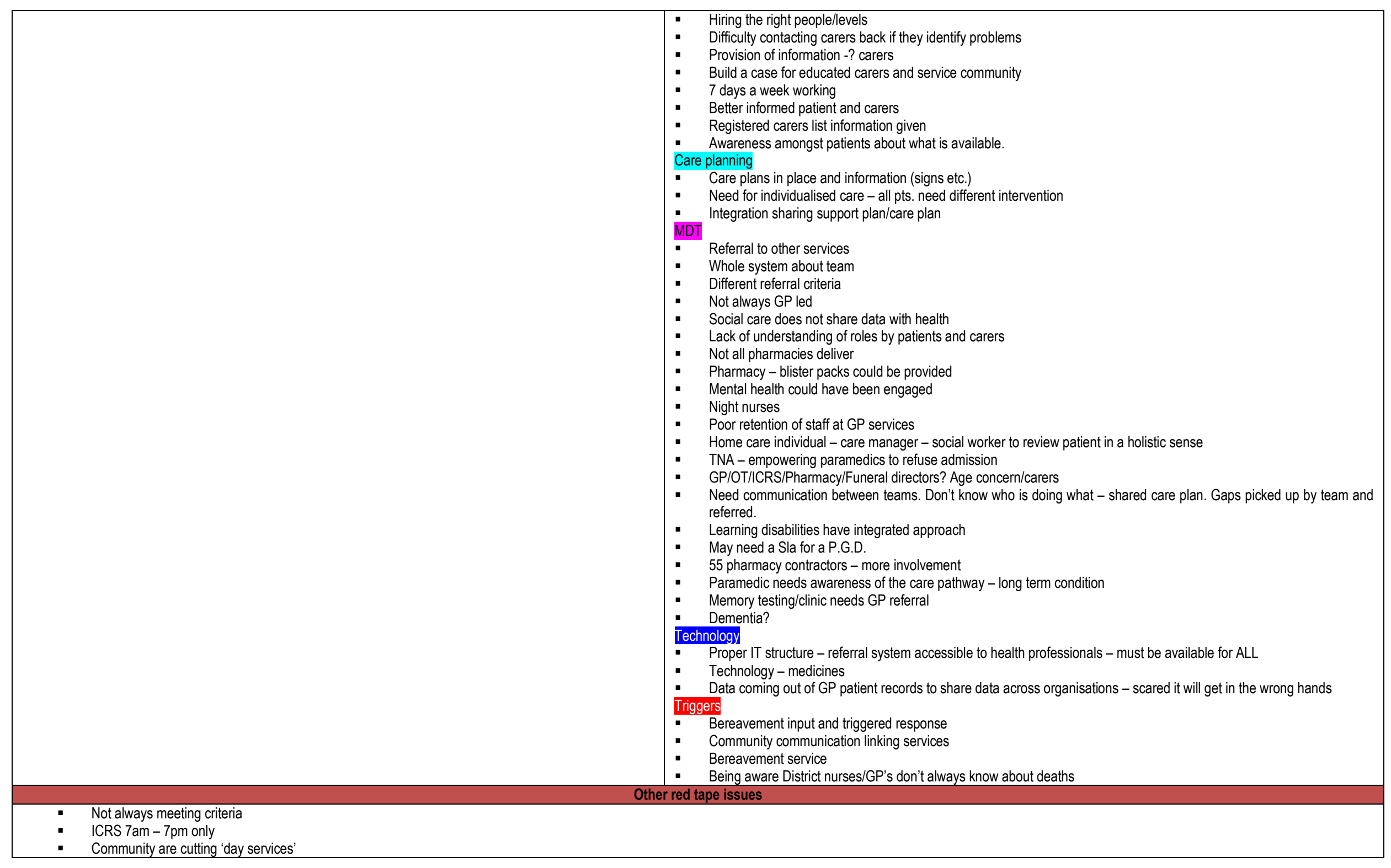




\section{Workshop 1 Participant attitudes towards the use of SqS pre and post-Questionnaire results}

The pre-questionnaire workshop revealed that very few attendees had any or much knowledge of the current system in which they worked (87\%). However, by the end of the workshop, this was improved vastly with $100 \%$ of attendees stating they had some or a lot of knowledge. $90 \%$ felt that their knowledge of the current system had improved with one respondent stating "Really useful to talk to other services in in the area, nice to put faces to names". The SqS approach was also highly rated with $100 \%$ of attendees stating it was beneficial to them, and $95 \%$ stating it was necessary to get the points across. One attendee stated "Stimulates thinking in a way written case study does not" and another "Very useful in stimulating the discussions and improves participation". Other things that attendees enjoyed about the workshop was "Ability to provide input which will (hopefully) bring real change and improve things in future" and "The scenarios were very interesting to see how others work". When asked what they would change about the day the majority of responses were practical in terms of room temperature and size, however one respondent stated "Information on how other areas are doing, whole systems or other similar projects are doing this. Also worth learning about their achievement so we don't reinvent the wheel!"

\section{Workshop 2 Participant attitudes towards the use of SqS pre and post-Questionnaire results}

The feedback from the second workshop also revealed that attendees felt they had an opportunity to raise questions, ideas and concerns (100\%), and $95 \%$ felt they had had the opportunity to make a useful contribution to the new model of care. $80 \%$ of attendees said their knowledge of the new model of care had improved with one attendee stating "Knowing different service available. MDT involvement, importance of other areas, finding the missing link". All attendees agreed that the simulation content and simulation as a focus for discussion was good and excellent with one attendee stating "Captures the needs of the client" and another "A good way/method of training". When asked how they felt about SqS as an approach, respondents stated "It is very helpful to see imagined solutions in practice. i.e. Raj - suggestions from workshop 1 didn't really work when we saw them in practice" and another "I thought the simulation idea really worked well as you could see clearly what would work and what doesn't work" as well as "I think it works well, because it enables you to see what approach is used and why it is working or not working." $95 \%$ of attendees at the second SqS workshop felt the Sequentially simulated scenarios helped them to visualize how the new model of care might work in practice "It did visualise the new model. I feel we have a lot of good services and systems in place. We channel them better". $100 \%$ said the SqS was beneficial to them and $90 \%$ said it was necessary to get the points across - "Helped get diverse audience to combine understanding". Overall, attendees felt the workshop was successful with one attendee stating "The enthusiasm and engagement of all the participants - tribute to the event that more people came for the second session" and "Simulations had a human element and a touch of humour. Helped whole integrated care appear real" as well as "Change in outcomes of simulation through integration and overall approach". The Clinical Commissioning Group lead stated "The data taken from the sequential simulation workshops has helped us to create an in-house operating model" 


\section{DISCUSSION}

This approach to engaging and co-designing solutions to new models of care with key stakeholders was received positively. The feedback from the workshop questionnaires revealed that attendees at both workshops strongly agreed that they had had an opportunity to contribute to all discussions and had plenty of opportunities to raise questions, concerns and ideas. The pre and post knowledge of current and new models of care was vastly improved in both workshops and the opportunity to information share and network was appreciated with the SqS approach breaking professionals barriers and identifying roles and services. The SqS's enabled attendees to visualize current services, as well as their own 'idealised' models in practice before any implementation process had been considered.

The process of using various data collection methods to capture as much of the discussions as possible, as well as the mixing of professionals into smaller facilitated groups, proved to be an effective way of engaging and involving front-line staff and patients in new system modeling. This data capturing and engaging process also enabled the formation of a final report that was available to the CCG and disseminated further, revealing stakeholder knowledge and understanding, their questions and concerns; as well as their valuable input into how to make an integrated system work at an operational level.

The atmosphere at both workshops was extremely positive and provided an opportunity to commence a culture shift from what can't be done to what can be done and how. Attendees felt that they were being listened to. This was further verified by seeing their discussions in an analysed format and the subsequent 'idealised' scenario generated. Further proof of this comes from the repeated requests of attendees to have copies of the final report.

In order to keep momentum, the workshops were held 8 days apart. This therefore required a lot of organization and a dedicated group of people to ensure consistency across both workshops. Skills in both simulation and research approaches were essential components to ensure authenticity and rigor; both integral to this remodeling approach.

\section{Limitations}

Due to the nature of the SqS model, the direct results of the remodelling data collected cannot be generalised. However, the concept itself is transferrable. Efforts were made to ensure all attendees had a 'voice'; this is apparent through the varied forms of data collection utilised.

The practicalities, manpower, and simulation and research skills required to undertake this collaborative healthcare remodelling approach is significant; and good design and planning essential. However, the benefits potentially generated in terms of breaking down professional barriers, culture shifting, design and evaluation of, as well as, buy in to new models of care is also significant and both should be considered when undertaking this approach.

\section{Conclusions}

The need and usefulness of this approach is apparent from the attendee's feedback, as well as the remodelling data co-generated. The SqS model approach enables this form of SqS to be tailored to local contexts ensuring it is transferrable in a bespoke manner. The systematic approach to data collection from the diverse ideas generated through the SqS also allows for a much needed 'ear' to those providing the solutions, as well as a legitimate and balanced perspective. 
Further studies of this approach are needed in order to understand the rich and complex benefits that SqS can provide in terms of collaboratively remodelling healthcare. Additional data is currently being collected and analysed by the authors.

\section{Acknowledgements}

The authors' would like to thank Shvaita Ralhan, Zinah Sorefan, Ana Rita Rodrigues, Laura Coates and Matt Gold for their help in undertaking the workshops, collecting and compiling its associated data.

Figures 3 and 4 are photos of participants simulating the described healthcare scenarios and not real patients or real clinical encounters.

\section{Funding}

This work has been supported by the North West London Integrated Care Pilot, Health Education North West London, Hounslow Clinical Commissioning Group and Imperial College Health Partners.

Competing interests: The authors declare no conflicts of interest. 


\section{References}

1. NHS England. Integrated Care and Support Pioneer Programme: Annual Report 2014.

Accessed on $1^{\text {st }}$ December 2015 from

http://www.local.gov.uk/documents/10180/6927502/Integrated+Care+Pioneer+Programme +Annual+Report+2014/76d562c3-4f7d-4169-91bc-69f7a9be481c

2. Nuffield Trust. An overview of integrated care in the NHS: What is integrated care? 2011. Accessed on $25^{\text {th }}$ November 2015 from:

http://www.nuffieldtrust.org.uk/sites/files/nuffield/publication/what is integrated care re search report june11 0.pdf

3. World Health Organisation. A glossary of terms for community of health care and services for older persons. 2004. Accessed on $24^{\text {th }}$ November 2015 from: http://www.who.int/kobe centre/ageing/ahp vol5 glossary.pdf

4. Beaufort B., \& Longest, Jr. Health Programme Management: From development through evaluation. San Francisco, John Wiley \& Sons. 2014:211-217

5. NHS England. TRANSFORMING PARTICIPATION IN HEALTH AND CARE 'The NHS belongs to us all'. 2013. Accessed on $25^{\text {th }}$ November 2015 from: https://www.england.nhs.uk/wpcontent/uploads/2013/09/trans-part-hc-guid1.pdf

6. Bourdieu P. The Logic of Practice. Cambridge: Polity Press. 1990: 112-120

7. Elliot, E., \& Williams G. Developing public sociology through health impact assessment. Sociology of Health \& Illness. 2008. Vol. 30 No. 7 pp. 1101-1116

8. Kneebone, R. Simulation, Safety and surgery. BMJ Quality \& Safety, 2010. 19: 47-52 doi:10.1136/qshc.2010.042424

9. Weldon, S-M., Ralhan, R., Paice, E., Kneebone, R., and Bello, F. Sequential simulation (SqS): an innovative approach to educating GP receptionists about integrated care via a patient journey. BioMed Central Journal Family Practice. 2015. 16: 109

10. Weldon, S-M., Ralhan, R., Paice, E., Kneebone, R., and Bello, F. Sequential simulation of a patient journey. The Clinical Teacher. 2016 (in-print)

11. Powell, P., Sorefan, Z., Hamilton, S., Kneebone, R., and Bello, F. Exploring Sequential Simulations potential as an educational tool in paediatrics. The Clinical Teacher. 2015. 12: 17

12. Weldon S-M, Coates L, Kneebone R and Bello F. (2014) Hounslow Whole System Integrated Model of Care Sequential Simulation (SqS) Workshops, Health Education North West London - Simulation: Is a New Approach Needed? Conference.

\section{Figure legends}

Figure 1. Workshop 1 attendance grouped by role (65 attendees)

Figure 2. Workshop 2 attendance grouped by role (93 attendees)

Figure 3. A diagram of Navneeta's 'current' Sequentially Simulated story

Figure 4. A diagram of Navneeta's Sequentially Simulated 'ideal' story 